\title{
Harmony: A Hand Wash Monitoring and Reminder System using Smart Watches
}

\author{
Md Abu Sayeed Mondol \\ Department of Computer Science \\ University of Virginia \\ Charlottesville, VA, USA \\ mm5gg@virginia.edu
}

\author{
John A. Stankovic \\ Department of Computer Science \\ University of Virginia \\ Charlottesville, VA, USA \\ stankovic@cs.virginia.edu
}

\begin{abstract}
Hand hygiene compliance is extremely important in hospitals, clinics and food businesses. Caregivers' compliance with hand hygiene is one of the most effective tools in preventing healthcare associated infections (HAIs) in hospitals and clinics. In food businesses, hand hygiene compliance is essential to prevent food contamination, and thus food borne illness. Washing hands properly is the cornerstone of hand hygiene. However, the hand wash compliance rate by the workers (care givers, waiters, chefs, food processors and so on) is not up to the mark. Monitoring hand wash compliance along with a reminder system increases the compliance rate significantly. Quality of a hand wash is also important which can be achieved by washing hands in accordance with standard guidelines. In this paper, we present Harmony, a hand wash monitoring and reminder system that monitors hand wash events and their quality, provides real time feedback, reminds the person of interest when he/she is required to wash hands, and stores related data in a server for further use. Worker worn smart watches are the key components of Harmony that can differentiate hand wash gestures from other gestures with an average accuracy of about $88 \%$. Harmony is robust, scalable, and easy to install, and it overcomes most of the problems of existing related systems.
\end{abstract}

\section{Categories and Subject Descriptors}

C.3 [SPECIAL PURPOSE AND APPLICATION

BASED SYSTEMS]: Real-time and embedded systems

\section{General Terms}

Design, Experimentation

\section{Keywords}

Hand Hygiene, Smart Watch

\section{INTRODUCTION}

Hand hygiene compliance by workers is extremely important in healthcare settings like hospitals and clinics, in food businesses like restaurants, groceries and food processing industries. It is one of the most effective tools in preventing healthcare associated infections (HAIs) in hospitals [5, $17,22]$. In the year of 2011 , the number of HAIs in acute care hospitals of USA was estimated to be 0.72 million, and deaths associated with HAIs were about 75 thousand [1]. The annual direct medical cost of HAIs to U.S. hospitals is estimated to be in the range of 28 to 45 billion US dollars [21]. The rates and risks in the low and middle income countries are significantly higher compared to the high-income or developed countries [4]. Hand hygiene of food workers is essential in preventing food contamination and food-borne illness. One study shows that food contaminations by food workers are responsible for about $89 \%$ of the food borne illness outbreaks [20].

Washing hands properly is the cornerstone of hand hygiene compliance. However, adherences with hand wash practices among health care workers are significantly low compared to the requirements, and depend upon a number of factors like demographic characteristics of the health care workers, accessibility of hygiene product supplies, workload, and individual cognitive factors [18]. Reminding workers to wash their hands when necessary is very effective for ensuring hand hygiene adherence [14]. It is important to identify an individual's hand wash practices for providing personalized feedback, and for ensuring accountability.

The quality of each episode of hand washes is also important, and can be achieved by complying with some standard guidelines like one provided by World Health Organization (WHO) [3]. The guideline is shown in figure 1. According to the guideline, proper hand washing procedure comprises of different ways of rubbing the hands to ensure that no area of the hands is missing. Most of the existing hand hygiene monitoring systems can detect hand wash events only, and lacks the capability of recognizing the quality.

It is very important for the hospital/business authorities as well as public health agencies to monitor and measure hand hygiene adherence by the workers, and to provide feedback to the stakeholders. Different methods exist for this purpose like surveys, self-reporting, direct observation by human observers, indirect observations based on product utilization, and automated monitoring systems. Surveys and self-reporting approaches require significant human effort. Results from these approaches are often incomplete, error prone, and biased. Direct observations by human observers 
require lots of human effort, and often result biased data and uncomfortable working environment. Indirect observation method estimates the number of hand wash events based on the utilization of hand wash products. This method requires less effort than direct observation. But it is limited in providing personal and temporal information, like who uses the products, and when. The duration and quality of a hand wash episode can not be determined using this approach. Automated monitoring systems require almost no human effort, and provide more accurate results. Though these automated systems can detect hand wash events, most of them cannot measure the duration and quality of the episodes. A vision based technique to monitor the quality of hand washes is presented in [11]. Cameras are placed on each sink to detect hand gestures. However, the system doesn't detect the person who is washing hands, and it also doesn't provide any reminder. Vision based techniques come with a number of limitations like privacy violation, environment dependent accuracy, and higher costs.

In this paper, we present Harmony, a smart watch based hand wash monitoring and reminder system that overcomes most of the limitations of the existing systems. Smart watches available today are Bluetooth enabled, and are enriched with different sensors like accelerometers and gyroscopes that can capture the hand motions. In our system, each worker wears a smart watch when he/she is on duty, and sensors of the watch are used to identify different gestures related to hand wash. The system identifies a worker by an identification code stored in the smart watch he/she wears. Quality of a hand wash is determined by the duration of the hand wash episode, and the presence of the different gestures as shown in figure 1. The required duration for each of the gestures is not specified in the standard. So, we only determine their presence or not in a hand wash. Our system provides reminder to a worker if he/she forgets to wash hands when required, or the quality of a hand wash is not up to the requirement. All the computations are carried out in the watches at runtime, and all the data related to hand hygiene compliance are automatically stored in a server. To our best knowledge, Harmony is the first complete system with all the features mentioned.

The contributions of this paper are as follows:

- A novel hand wash monitoring and reminder system that is designed efficiently to run on resource limited smart watches.

- Evaluation is performed on two datasets where data is collected from 16 subjects including males and females.

- Using a short and simple training period, the system achieves accuracy of as high as $97 \%$ with an average of $88 \%$ for person dependent hand wash gesture detection.

- The evaluation also shows that person independent gesture classification does not work well, and that use of a gyroscope is not required.

- The system is robust, scalable and easy to install, and it overcomes most of the limitations of existing systems.

The rest of the paper is organized as follows. Section 2 describes the related work. Section 3 and 4 provide the details

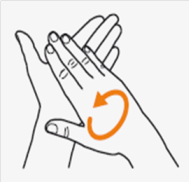

(1)

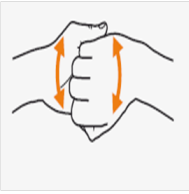

(4)

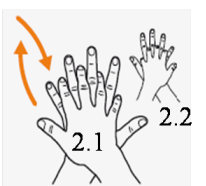

(2)

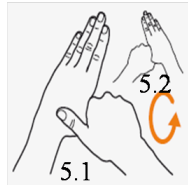

(5)

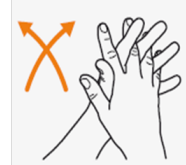

(3)

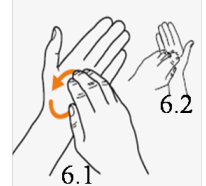

(6)
Figure 1: Hand wash guidelines by $\mathrm{WHO}^{[3]}$

of the system. Experimental results and analysis are presented in Section 5, followed by a discussion on robustness and scalability of the system in Section 6. A brief discussion on several issues related to the system, and future directions are presented in Section 7. Finally, Section 8 concludes the paper.

\section{RELATED WORK}

Due to the enormous importance of monitoring and ensuring hand hygiene compliances, the issue of developing efficient and effective systems to serve this purpose has received significant attention from the research community. A smart phone based application called iScrub helps human observers to collect and manage data easily [9]. The application is customizable, and an observer can export data in commaseparated-value format to e-mail addresses of his/her choice. In [7], a closed surveillance camera is positioned at the entrance to a surgical ward, and video data are collected. A pre-installed alcohol gel dispenser is available at the entrance of the ward. Entrants to the ward are supposed to use the alcohol gel before they enter into the ward. Footage is reviewed by human observers to monitor compliance by the entrants. All these systems involve one or more human observers, and require lots of human effort and related costs. They often result in biased data, and uncomfortable working environments.

An automated dispenser monitoring system is presented in [10] to count the hand wash episodes in hospitals. Pressure sensors are used to detect depression in wall-mounted soap and alcohol gel dispensers. A single press on the dispenser is then associated with a single hand-hygiene episode. The system generates false positives when a person dispenses multiple times during a single episode. It also cannot identify the workers, and the quality of an episode. The system has no capability to provide notifications or reminders. In [8], a system to detect the use of alcohol based sanitizer is presented. An alcohol sensor is used to detect the vaporization that comes from the hand after a use of the sanitizer. The system does not work when hands are washed using soap and water. It does not provide any reminder.

A system consisting of small credit card sized devices is described in [19]. The devices are programmed to perform three different roles called badges, beacons, and triggers. The badges are worn by the health workers, the beacons are placed in the patient rooms, and the triggers are attached to the dispensers. The devices can communicate using a 
protocol for personal area network (PAN). Another automated hand hygiene documentation and reminder system is described in [13]. Pressure or vibrator sensors are attached to the dispensers to detect dispensing of soap or alcohol gels. Upon detection of a dispensing event, it enables a passive infrared sensor to detect human hands, and then an alcohol sensor is used to identify the use of alcohol based sanitizer. Ultrasonic hotspots are created in wash zones and patient bed zones, and each healthcare worker wears a wireless tag as a badge that detects the ultrasonic hotspots. These systems cannot detect the worker who performed a hand wash when multiple persons are present in a wash zone at a time, and cannot detect the quality of the hand wash episodes.

Most of the automated systems cannot measure the quality of a hand wash. A vision based technique to measure the quality of hand washes is presented in [11]. Each sink is equipped with a camera, and gestures of the hands are analyzed to measure the quality. To discriminate between different types of hand gestures, a support vector machine (SVM) classification technique is applied to the features extracted from each frame. It does not identify the person performing the hand wash, and does not provide any reminder. Also it requires one camera for each of the sink or dispenser area where hand hygiene is performed. Any camera based approach comes with a number of limitations and problems. Deploying cameras in washroom areas is very uncomfortable for the users as well as raises privacy issues. Also for easy access, sanitizer dispensers are often kept in the areas where patients are located. Placing cameras in these areas is threatening to the privacy of the patients [16]. The required number of cameras for settings like hospitals is generally very large; and massive computing and communication resources are required to collect and process the data. Such systems are very expensive to install and maintain. The performance of vision based system also depends on environmental factors like lighting and decoration of the surroundings.

\section{SYSTEM DESCRIPTION}

The main components of Harmony are smart watches, Bluetooth beacons, Bluetooth enabled liquid dispensers, relays and a server. A relay in Harmony is a Bluetooth enabled device that can communicate with both the smart watches and the server. Most of he smart watches available today do not have the capability to communicate through Wi-Fi/Cell networks, and so relays are used for the communication between the server and the watches. However, the relays are not needed if the watches can communicate with the server directly.

The dispensers in Harmony are Bluetooth enabled. A dispenser broadcasts advertisements using Bluetooth when liquid is dispensed from it. The range of the Bluetooth transmitter of the dispensers is kept short, usually within one meter, and the area covered by the range is called a dispenser zone.

Bluetooth beacons are small devices that periodically broadcast advertisements. These devices are placed in areas where it is required to detect whether a worker has washed hands recently. For example, they may be placed at the entry or exit of patient rooms in hospitals. In restaurants and food processing industries, they may be placed at the entry of food processing zones, and at the exit of workers' toilets. The transmission power of the beacons is configurable, and

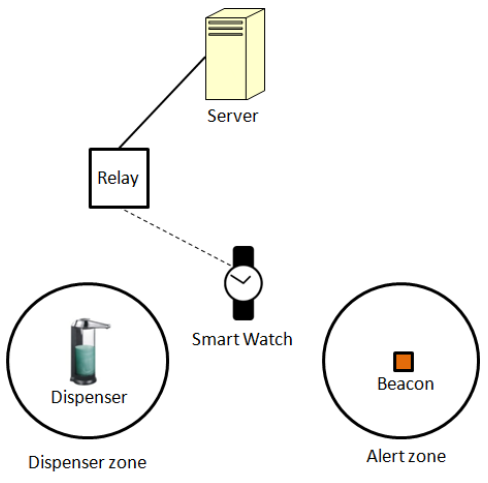

Figure 2: Components of Harmony

it is determined based on the area coverage required. The area covered by a beacon is called an alert zone. All the computations are carried out in the watches, and resulting data are saved in a server to be used for useful reports. The components of Harmony are shown in figure 2 .

Harmony requires each of the workers to wear a smart watch while they are on duty. Each time a worker washes hands, the smart watch worn by the worker detects the time, the duration, and the quality of the hand wash episode, and saves this information. When the worker enters into an alert zone, the watch checks whether he/she has washed hands recently. If not, it reminds the worker with alerts. Alerts are also generated if the worker has washed hands, but the quality of the wash is not up to the requirement of that area. Alerts are provided in forms of vibration, beeps, or both along with displaying information on the watch. The work flow in Harmony is shown in figure 3.

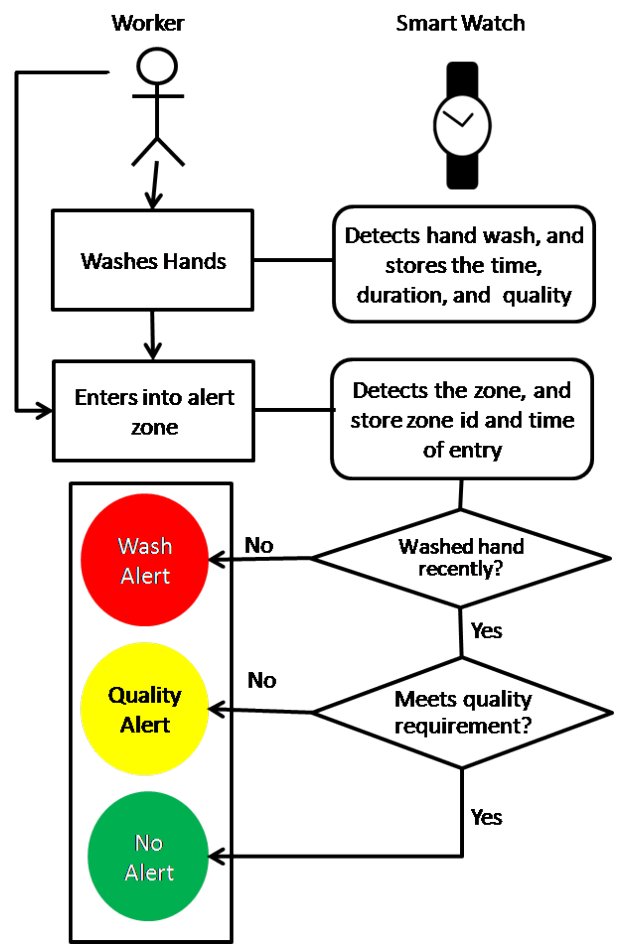

Figure 3: Workflow in Harmony 


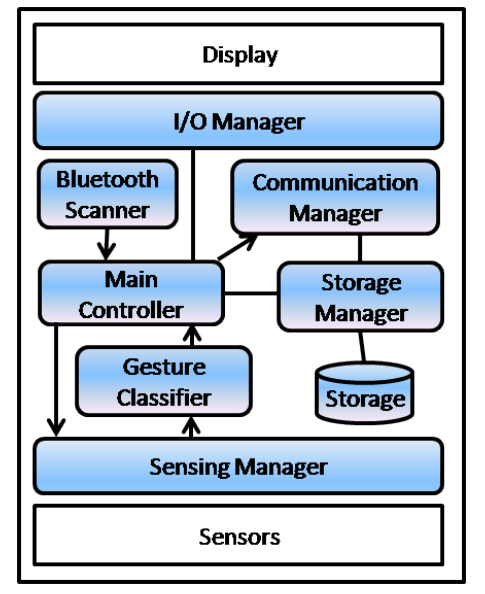

Figure 4: Watch App Architecture

A smart watch app is installed in every watch of our system to perform the required functionalities. The architecture of the app is modular as shown in figure 4. Bluetooth Scanner scans for Bluetooth advertisements. It only accepts the advertisements from the dispensers, the beacons and the relays of Harmony, and ignores the advertisements from the devices that are not part of the system. The accepted advertisement data along with the received signal strength indication (RSSI) of the advertisement is sent to Main Controller which is responsible for controlling the hand gesture recognition process, reminders, and display. When hand wash gestures need to be recognized, the Main Controller enables the Sensing Manager which collects raw sensor data. Collected data are transferred to the Gesture Classifier that processes and analyzes the data, and recognizes relevant gestures. To save energy, the Sensing Manager is disabled when gesture recognition is not required. The I/O Manager is responsible for taking user inputs, managing the display, and generating alerts.

Scanning for Bluetooth signals consumes significant power. To save energy of the watches, the Bluetooth Scanner module should run with a low duty cycle. However, a low duty cycle may cause late hearing of signals from beacons and dispensers, resulting in a delayed reminder, or delay in starting the hand wash recognition process. Harmony uses an adaptive duty cycle approach for the Bluetooth signal scan. The distance of a watch from a beacon is estimated using the received signal strength indication (RSSI) of the signals, and the transmission power of the beacon. When the location of a worker is estimated to be near an alert or dispenser zone, the duty cycle is increased. When the worker moves away from the zone, the duty cycle is decreased. The adaptive approach saves energy of the watches while ensures that signals from beacons or dispensers are received in a timely manner. The approximate distance, not the exact location, of a user from a beacon is required for providing alerts to the user. Though RSSI values fluctuate depending upon factors like environmental noise and obstacles, the distance can be approximated since the radius of a alert zone is small and there is a line of sight communication between a watch and a beacon when the watch is in the alert zone.

The Main Controller stores different information related to hand wash compliance in the storage of the watch through the Storage Manager that facilitates data management and manipulation operations like insert, retrieve, delete, and update. The Communication Manager transfers data to the server through relays.

\section{DESIGN OF HARMONY}

The three tasks that Harmony performs are hand wash recognition, providing reminders (alerts), and data logging. Detail approaches for each of the tasks are described in the following subsections.

\subsection{Hand Wash Recognition}

The accelerometer and gyroscope of the smart watches are used to recognize different gestures related to hand washing. To save energy, a smart watch runs the hand gesture recognition process only for small time periods when it gets signals from a dispenser. This approach also reduces false positives compared to a continuously running process.

\subsubsection{Starting and Stopping Recognition Process}

When a worker dispenses soap/sanitizer, the dispenser broadcasts advertisements. Upon hearing the advertisement, a watch starts recognizing hand gestures, and continues until hand washing is finished. This approach ensures that hand wash gestures without using soap or sanitizer are not counted toward a hand wash event. The time, the duration, and the quality of each hand wash episode along with the identification number of the dispenser are stored in the watch. Duration and quality information are also displayed on the watch screen.

A watch may hear an advertisement from a dispenser even if the worker wearing the watch doesn't trigger it. This happens when multiple workers are present in a dispenser zone during a dispense event. Some of the possible such scenarios are given below.

- In most washrooms, multiple sinks/dispensers are placed closely. So, when one person dispenses soap, there may be another person in the area whose watch hears the dispenser signal. The other person may already be washing hands, or may have just finished washing hands, or may be going to dispense soap.

- A worker dispenses sanitizer from a dispenser while another caregiver is present in, or passes by the area. The watch of the second caregiver may hear the dispenser advertisement.

So, when a watch receives an advertisement from a dispenser, either the worker wearing the watch or any other person may trigger the dispenser. Figure 5 shows the mechanism of how a watch works when it gets an advertisement from a dispenser. The figure also depicts the process of starting and stopping the hand wash recognition process.

If an advertisement from a dispenser is received when the gesture recognition process is already running in the watch, the advertisement is ignored. Otherwise the watch starts the gesture recognition process upon hearing the advertisement. If hand wash gestures are not detected within a certain period of time after starting of the recognition process, the watch stops the process considering that the worker wearing the watch didn't trigger the dispenser. This is the time period within which a worker is expected to start washing hands after the soap/sanitizer is dispensed, and it is called 
the Start Time-out Period $(S T P)$ in our system. If hand wash gestures are detected within $S T P$, the recognition process is continued until the hand wash is finished. Generally, workers start washing hands immediately after dispensing soap/sanitizer. However, there may be a delay sometimes, specially for some settings where the dispenser and sinks are far apart. The STP needs to be long enough so that most of the delays can be incorporated. On the other hand, a longer $S T P$ may result in more power consumption of the watch. In Harmony, the $S T P$ is configurable, and it is set to 20 seconds by default.

A worker may pause washing hands for a short duration during a hand wash episode. So, the hand wash recognition process is not stopped as soon as the hand wash gestures are not found. Rather the process is stopped if hand wash gestures are not found for a specific time period which is called the End Time-out Period $(E T P)$ in our system. The $E T P$ is also configurable, and set to 20 seconds by default. Due to recognition errors, some gestures during a hand wash may be recognized as non hand washing, and vice versa. The duration of the errors are usually very short, and depends on the precision and recall of the gesture recognition model. Figure 6 shows an example of errors in hand wash gesture detection. These errors are excluded from STP and ETP calculations.

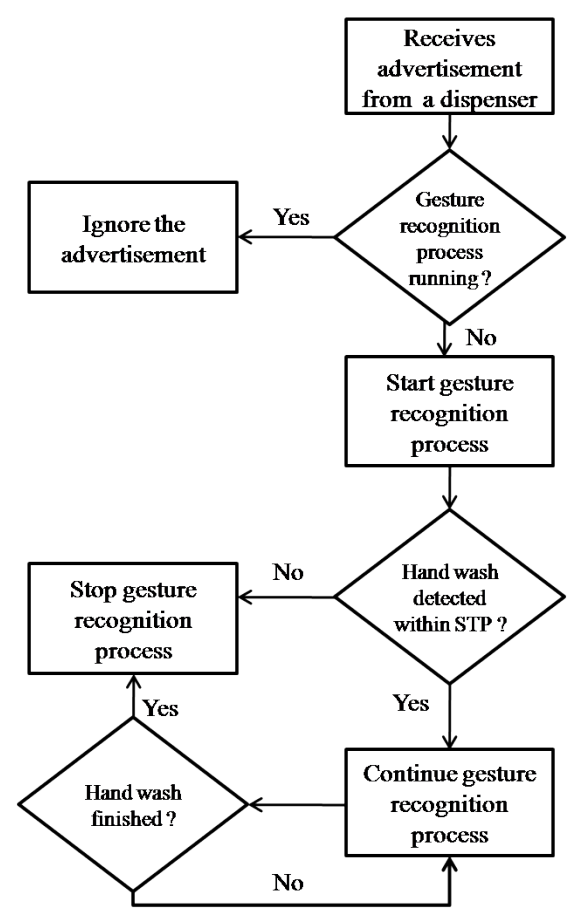

Figure 5: Mechanism for starting and stopping the gesture recognition process in a watch

\subsubsection{Discriminating Hand Gestures}

To discriminate different hand gestures, data from sensors are grouped into small time windows, and classification is done on each of these windows. In Harmony, hand wash gestures are discriminated in two phases. In the first phase, hand wash gestures are differentiated from non hand wash gestures, and if any window is recognized as of hand wash, the window is classified as any of the poses (figure 1) or as

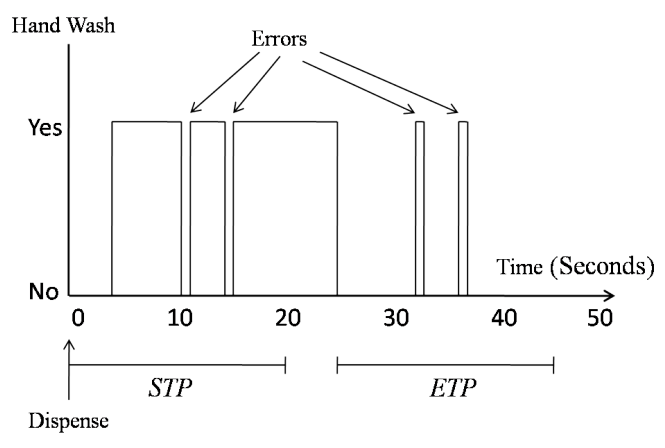

Figure 6: $S T P$ and $E T P$ in a hand wash episode

others in the second phase. Two different classification models are used in the two phases. Since it requires significant computation and memory in building efficient and effective classification models, model building process is carried out in servers. The models are then used in the watch to classify gestures at runtime.

\section{Building Classification Models}

Annotated linear acceleration, gravity, and gyroscope signals for different hand gestures are collected from smart watches, and they are processed and analyzed in servers. All signals are annotated as hand wash or non hand wash. Each hand wash signal is then annotated as any of the gestures as shown in figure 1 or as others.

Features are extracted on one second windows from the time series signals with 0.2 second window sliding. Since the processing and memory capacity of the smart watches are very limited, memory and computationally efficient features and classification techniques need to be used. Extraction of time domain features is usually less computation intensive compared to frequency domain features [15]. In Harmony, we use time domain features only. As mentioned before, two different classification models are generated. One binary classification model is built to detect each window as hand wash or not. Another multi-class classification model is built that classifies each hand wash window as any of the poses of figure 1 or as others. These models are called the Wash Detection Model (WDM) and the Pose Recognition Model $(P R M)$, respectively, in our system.

\section{Runtime Classification}

Harmony recognizes hand wash gestures in real time. Time series sensor signals are grouped into one second windows, and features are extracted on each window. At first, features required for $W D M$ are computed, and the model is applied to determine whether the window is hand wash or not. If the window is detected as hand wash, features for $P R M$ are computed, and the model is used to classify the window as any of the poses of figure 1 or as others. Since $P R M$ is applied on the same window for $W D M$, features for $P R M$ that are present in the feature set of $W D M$ are not recomputed. The runtime classification process is depicted in figure 7 . In the figure, $F_{W}$ and $F_{P}$ denote the feature set for $W D M$ and $P R M$, respectively.

\subsection{Providing Reminders}

As shown in figure 3 , Harmony provides two types of 


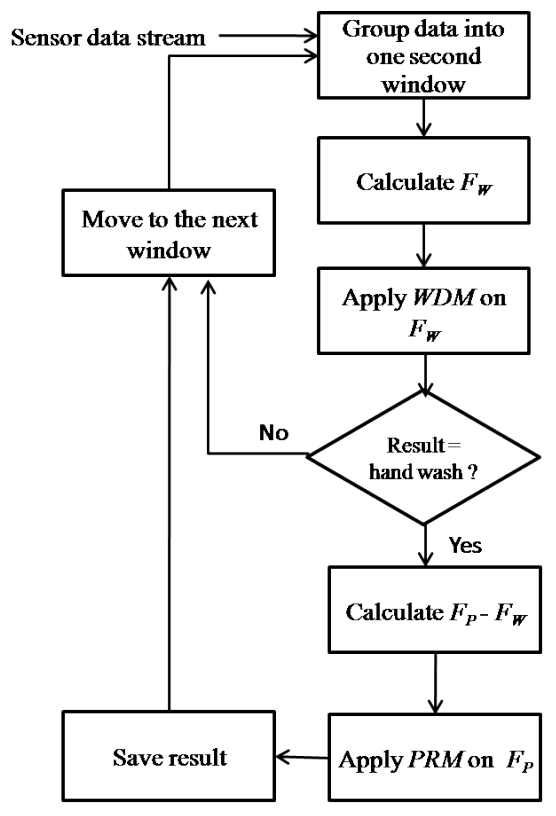

Figure 7: Runtime classification process

alerts/reminders to the workers, hand wash alert and quality alert. Along with vibration and beeps, the type of alert is displayed on the watch screen.

\subsubsection{Hand Wash Alert}

To provide a hand wash alert, it is necessary to determine whether the worker has washed hands recently. How recently a worker should wash hands before entering an alert zone depends on factors like the worker's psychophysical conditions, and the distances between the alert zone and the near by dispenser zones. The recentness time of a zone is included in the advertisement of the corresponding beacon, and the default value is 1 minute. This value can be configured separately for each of the beacon in our system. To address the psychophysical condition of a worker, a worker dependent factor is multiplied with the recentness time of the zone. The factor is set in the app of the worker's watch, and its default value 1 . For example, this factor value can be set to 1.5 for a physically impaired worker who moves slowly so that the worker gets more time to move from the dispenser zone to the alert zone.

\subsubsection{Quality Alert}

To determine whether a quality alert needs to be provided to a worker entering into an alert zone, the watch needs to know the quality requirement of the zone, and the quality of the recent hand wash episode of the worker. In Harmony, quality of a hand wash is determined by the duration of a hand wash episode, and the presence of different poses (figure 1) in the episode. The duration, and the presence of the poses for the last hand wash episode of the worker is available in the watch as these data are saved whenever a worker finishes washing hands. The advertisement packet from a beacon includes the zone id, and the quality requirement for the zone. The watch checks whether the quality of the last hand wash by the worker meets the requirement of the zone. If it doesn't, alert is provided to the worker through vibration and/or beeps. Also, the watch displays the duration, and which of the gestures from figure 1 are present in the last hand wash, along with the duration and poses required for the zone.

\subsection{Data Logging}

As mentioned earlier, data related to hand wash compliance of the worker are stored in the watch temporarily. The relays periodically broadcast advertisements. Whenever a watch discovers a relay and it needs to send data to the server, the watch establishes a connection with the relay, and data are transferred from the watch to the relay that eventually sends the data to a server. The watch deletes all the transferred data from its storage except the one from the last hand wash episode which is used for alert generation. The relays are not needed if the watch can transfer data directly to the server using technologies like Wi-Fi.

Relays may be mobile or installed at some fixed places. The watches need to be in proximity of a relay for some time duration so that the data transfer happens. This can be done at the end of the working period of a worker, or at any other suitable time. There may be one or more relays in the system. Even smart phones can be used as relays.

\section{EXPERIMENTS}

We collect ground truth data using a smart watch for different hand gestures. The data are then processed and analyzed in a server to build and evaluate the classification models. Battery lifespan of the watch is analyzed for different scenarios. In the experiment, we use Samsung Gear Live, an Android powered smart watch that is Bluetooth enabled, and is enriched with different sensors including an accelerometer, a gyroscope, a magnetometer, and a microphone. We have used the accelerometer and the gyroscope sensors in the experiments.

\subsection{Ground Truth Data Collection}

The accelerometer and gyroscope available in the smart watches are tri-axial. Linear acceleration, force of gravity, and rotation rate along all the axes of the watch are collected at $50 \mathrm{~Hz}$ for different hand gestures as described below.

1. Rubbing hands according to the different ways recommended by WHO (figure 1). Each of the ways are performed in a single episode, and annotated separately.

2. Rubbing hands in other ways not in one of the ways mentioned in 1 .

3. Washing hands in any way including WHO recommended ways of hand rubbing. Here the gestures for different ways of hand rubbing are not separated, and the hand wash is annotated as a single episode.

4. Different non hand wash gestures including (but not limited to) dry hands with towel or tissue paper, walking, drinking and random gestures.

Two sets of data have been collected. One set, called Dataset $1(D S 1)$, contains data from 9 subjects $(5$ males and 4 females, all graduate students) with age range from 25 to 32 years. Another set, called Dataset 2 (DS2), contains data from 7 subjects ( 4 males and 3 females) with age range from 17 to 36 years. The subjects from the two datasets are mutually exclusive. For Dataset 1, data for different 
Table 1: Duration of ground truth data

\begin{tabular}{|c|c|c|c|}
\hline \multirow{2}{*}{ SL } & \multirow{2}{*}{ Gesture } & \multicolumn{2}{|c|}{ Duration (sec) } \\
\hline & & DS 1 & DS 2 \\
\hline 1 & Handwash: pose 1 & 10 & 10 \\
\hline \multirow{2}{*}{2} & Handwash: pose 2.1 & \multirow{2}{*}{10} & 10 \\
\hline & Handwash: pose 2.2 & & 10 \\
\hline 3 & Handwash: pose 3 & 10 & 10 \\
\hline 4 & Handwash: pose 4 & 10 & 10 \\
\hline \multirow{2}{*}{5} & Handwash: pose 5.1 & \multirow{2}{*}{10} & 10 \\
\hline & Handwash: pose 5.2 & & 10 \\
\hline \multirow{2}{*}{6} & Handwash: pose 6.1 & \multirow{2}{*}{10} & 10 \\
\hline & Handwash: pose 6.2 & & 10 \\
\hline 7 & Handwash: pose others & 10 & 10 \\
\hline 8 & Handwash: all & 40 & 40 \\
\hline 9 & Non handwash & 110 & 140 \\
\hline \multicolumn{2}{|c|}{$\begin{array}{l}\text { Subtotal (per hand per sub- } \\
\text { ject one episode) }\end{array}$} & 220 & 280 \\
\hline \multicolumn{2}{|c|}{$\begin{array}{l}\text { Subtotal (per hand per sub- } \\
\text { ject) }\end{array}$} & $\begin{array}{l}220 \times 1 \\
=220\end{array}$ & $\begin{array}{l}280 \times 5 \\
=1400\end{array}$ \\
\hline \multicolumn{2}{|c|}{ Subtotal (per subject) } & $\begin{array}{l}220 \times 2 \\
=440\end{array}$ & $\begin{array}{l}1400 \times 2 \\
=2800\end{array}$ \\
\hline \multicolumn{2}{|c|}{ Total } & $\begin{array}{l}440 \times 9 \\
=3960\end{array}$ & $\begin{array}{l}2800 \times 7 \\
=19600\end{array}$ \\
\hline
\end{tabular}

hand gestures, as listed above, have been collected once for both left and right hands from each of the 9 subjects. For Dataset 2, data has been collected for five times (episodes) from each of the 9 subjects. In figure 1, there are nine different gestures, considering that each of the 2nd, 5th and 6 th ways of hand rubbing comprises two different gestures. For Dataset 2, these nine gestures are annotated separately. However, for Dataset 1, sub-gestures of the 2nd, 5th and 6th ways of hand rubbing are not annotated separately, resulting six different gestures from figure 1. The details and total duration of the datasets are shown in table 1

\subsection{Feature Extraction and Classification}

As all the computations in Harmony are carried out in the watch, a small device with limited resources, the feature extraction and classification techniques need to be less resource consuming. For classification, we use a decision tree technique which is less computing intensive, but provides reasonable accuracy. Methods like Hidden Markov Model (HMM) usually require significant computations, and so they are not used in our system. Time domain features, namely mean, variance, root mean squares, median, first quartile, third quartile and covariance, are used. Data from sensors are segmented into one second windows with 0.2 second window sliding. Features are extracted on a window for each of the axes of the sensors, resulting 21 features per window for each of linear acceleration, gravity and gyroscope. The Forward Sequential Feature Selection method with 10 fold cross validation is used to find the best features for each of the classification models. For example, nine features are selected for the personalized model for subject 1 from Dataset 2 when the first episode of data is kept out. The features are variance (X axis, Linear Acceleration), root mean square ( $Y$ axis, Linear Acceleration), variance (Z axis, Linear Acceleration), root mean square (Y axis, Gravity), third quartile ( $\mathrm{Z}$ axis, Gravity), covariance (Z-X axes, Gravity), third quartile (X axis, Gyroscope), root mean square ( $\mathrm{Y}$ axis, Gyroscope) and first quartile ( $\mathrm{Z}$ axis, Gyroscope).

As mentioned earlier, two different classification models are required in our system, one for detecting hand wash gestures, and another for recognizing different poses of hand rubbing. For hand wash detection, all hand wash instances (1-8 from table 1) are labeled as positive class, and non hand wash instances (9 from table 1) are labeled as negative class. For pose recognition, the labeled instances for different poses (1-7 in table 1) are used. To evaluate the impact of the gyroscope (rotation rate) for hand wash gesture recognition, two feature sets are used. One set includes the features of linear acceleration and gravity only, while the other includes gyroscope features in addition to the features of linear acceleration and gravity. We call the feature sets Lacc-Grav and Lacc-Grav-Gyro (Linear Acceleration, Gravity, Gyroscope), respectively. Classification models for left hand and right hand data are built and tested separately using the data from the corresponding hand. The accuracy results for left hand and right hand are not significantly different, and so in practice a user can wear the watch in any hand of his/her preference. Due to space limitations, we have shown only the average accuracy of both of the hands.

In the experiments, both person-independent and persondependent classification models have been evaluated. For the person-independent case, data of a subject are used to test the models built from data of other subjects. On the other hand, for the person-dependent case, data from the same person is used for training and testing. We have evaluated person-independent classification on both Dataset 1 and Dataset 2. Person dependent evaluation has been performed on Dataset 2 only where data of each episode of each subject is used to test the model built from other episodes of the same subject. Then, results for the five episodes are averaged to get the accuracy for each person. Since there is only one episode of data for each subject in Dataset 1 , this dataset isn't used for person-dependent evaluation. Accuracy in our system is defined as:

$$
\text { Accuracy }=\frac{\text { Number of correctly classified instances }}{\text { Total number of instances }}
$$

\subsection{Classification Result Analysis}

The accuracies for person-independent classification are shown in figure 8. Results show that hand wash detection accuracy is reasonable (about $85 \%$ ) while pose recognition accuracy is poor (about $55 \%$ ). The pose recognition requires differentiating between 7 and 10 different classes for Dataset 1 and Dataset 2, respectively. Similarity of hand gestures among some classes, and inter-person variability in performing hand washes are the reasons behind poor performance of pose recognition. Results show that the accuracy increase is not significant for hand wash gesture recognition when the gyroscope is used in addition to linear acceleration and gravity. As linear acceleration along different axes captures the rotation rate to some extent, the gyroscope data helps a little in differentiating hand gestures when used with linear acceleration and gravity.

The average accuracies for person-dependent hand wash detection and pose recognition are shown in figure 9 and 10 , respectively. Here we see that accuracies for most of the subjects (5 out of 7 ) are good. However, the performances of some subjects are very poor, specially for pose recognition, while those of other subjects are reasonable (80 to 90 


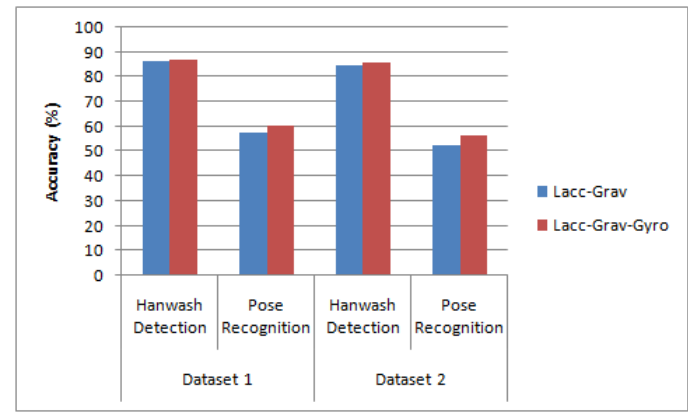

Figure 8: Accuracies for person-independent classification

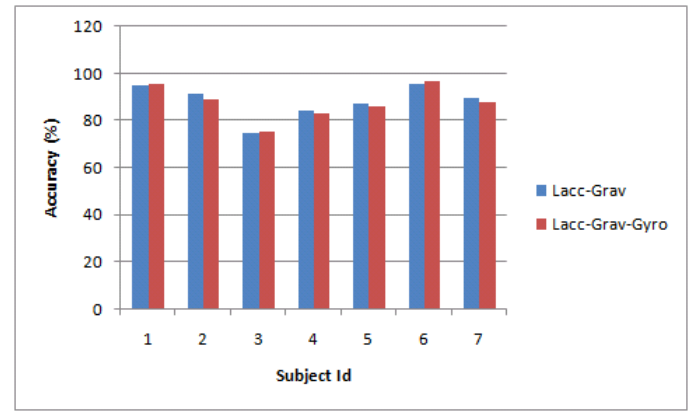

Figure 9: Accuracies for person-dependent hand wash detection

percent on average). One reason for poor performance of some subjects may be that they washed their hands in more inconsistent ways resulting more variability among different episodes of hand washes. Since subjects participated in the experiment are not professional caregivers or food workers, and they haven't washed hands before according to the WHO recommended ways, some of them are very likely to be inconsistent in performing different ways of hand rubbing.

In light of the experimental results we argue that personindependent classification should not be used if standard ways of hand rubbing (as shown in 1) needs to be differentiated, as the accuracy is very poor. Though participants in the experiment are not professionals, it is also very likely that each professional caregiver or worker has his/her own style of following the hand wash guidelines. On the other hand, professionals are more likely to be consistent in their style of washing hands compared to the participants in our experiment. So, person-dependent models should be deployed specially when different poses needs to be recognized with reasonable accuracy. If only hand wash events and their duration needs to be detected, person-independent model works with reasonable performance (about $85 \%$ accuracy), though person-dependent models perform better on average (about $88 \%$ on average, more than $90 \%$ for most of the subjects). Results also show that using rotation rate in addition to linear acceleration and gravity results in insignificant improvement.

\subsection{Battery Lifespan Analysis}

Smart watches run on rechargeable batteries, and different watches come with different battery capacity and lifespan. The battery capacity of the watch used in our experiment is $300 \mathrm{mAh}$. In this experiment we measured the battery lifespan of the watch for different scenarios. The typical

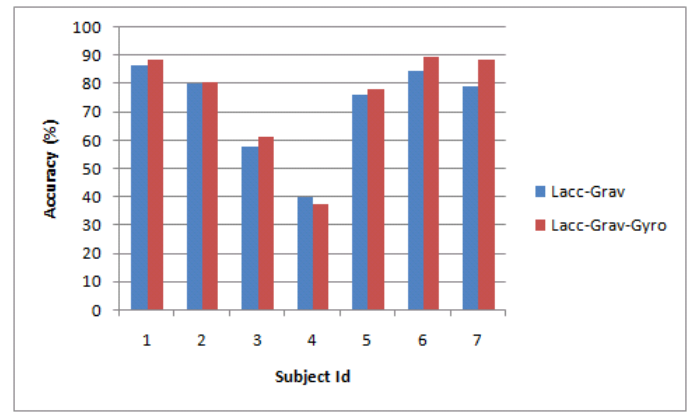

Figure 10: Accuracies for person-dependent pose recognition

lifespan of the watch used in this experiment is about 42 hours when the watch is used for time display only, with no other apps running.

The consumption of battery life per minute by the classification process is shown in figure 12, and it doesn't include battery consumption by other modules or apps. Results show that more battery power is consumed when the gyroscope is used in addition to linear acceleration and gravity. However, the classification process runs only when the watch gets advertisements from a dispenser, and it stops after the hand wash is finished. So, the total duration this process runs is typically very small compared to the total working period of the workers, and total energy consumption by this process is low. For example, if the process runs for total 25 minutes, and even if the gyroscope is used, it will consume only about $5 \%$ of the battery life of the watch.

The average battery lifespans for different duty cycles with one second active periods of the Bluetooth Scanner are shown in figure 11. The lifespans are due to the Bluetooth Scanner only, with no other module or apps running. Commercially off the shelf beacons like [12] can be configured to broadcast advertisement several times per second. So, a one second active period of the duty cycle is sufficient to detect any signal and it's strength from the beacons. Result shows that the watch runs for about 10 hours for $25 \%$ duty cycle (one second active for every four second time period). As our system uses an adaptive duty cycle, the average duty cycle should be smaller, resulting greater battery lifespan.

In our experiment we use a watch with battery capacity of $300 \mathrm{mAh}$. However, smart watches with $420 \mathrm{mAh}$ battery capacity are available now [2], and watches with more battery capacity are very likely in near future. The battery lifespan would be more as the battery capacity increases. For example, the capacity of a $420 \mathrm{mAh}$ battery is 1.4 times compared to a battery of $300 \mathrm{mAh}$, and so, the lifetime for $25 \%$ duty cycle with $420 \mathrm{mAh}$ capacity should be about 14 hours, 1.4 times for that with a $300 \mathrm{mAH}$ battery. Since hand wash compliance of a worker needs to be monitored only during his/her work hours, currently available smart watches can be used for a typical daily working period without recharging if the watch is charged sufficiently before use.

\section{SYSTEM PROPERTIES}

\subsection{Robustness}

Harmony is designed to work effectively in different scenarios. Though the robustness of the system is not tested through real deployment, its robustness is asserted from how 


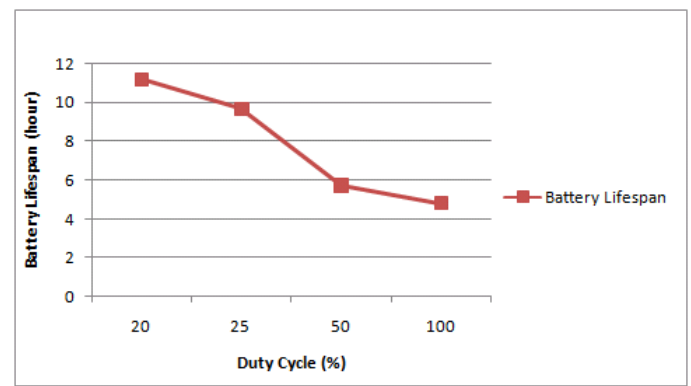

Figure 11: Average battery lifespan of the smart watch when Bluetooth Scanner runs

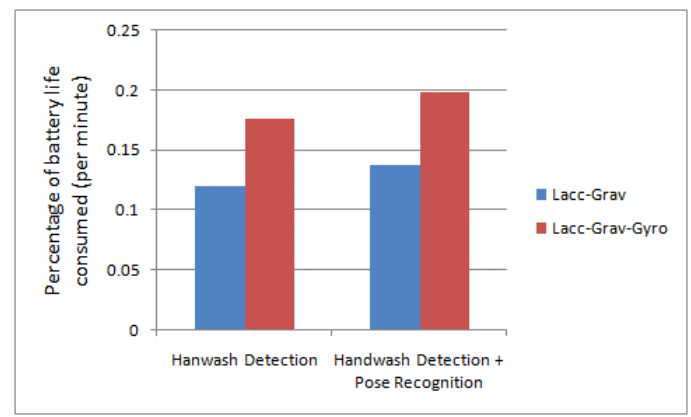

Figure 12: Average battery life consumption by the runtime classification processes

it works. One limitation of many existing automated systems is that they cannot identify the worker who performs a hand wash when multiple workers are present in the dispenser zone at the time soap/sanitizer is dispensed. Whenever a worker worn device, like a badge or a band, receives a dispense signal from a dispenser, the systems consider that the worker wearing the device performs a hand wash. So, all the workers present in the dispenser zone are considered to be washing hands though only one worker triggers the dispenser. In Harmony, when a watch receives an advertisement from a dispenser, it doesn't consider the worker wearing the watch to be washing hands if hand wash gestures are not found. So, Harmony can effectively detect the worker performing a hand wash even if multiple workers are present in a dispenser zone during a dispense event.

One problem of the existing vision based systems is that they may not perform well when sanitizer is used for washing hands. Since no sink/water is needed for washing hands using a sanitizer, a worker may move away from the dispenser while washing hands, or his hands may be out of focus of the cameras during a hand wash. In such cases, only a part of the hand wash episode is captured, and the quality of the whole episode cannot be determined. Since hand motions are used to recognize different hand gestures in Harmony, the presence of different poses and the duration of a hand wash episode can be determined even if the worker moves while washing hands.

The workers may perform some non hand wash gestures that are nearly similar to hand wash. In Harmony, the gesture recognition process in a watch runs for a short period of time only when the watch receives an advertisement from a dispenser. As the recognition process is disabled for most of the time, and short duration errors are smoothed out (figure
6 ), the rate of false positives resulting from non hand wash gestures is nearly zero in Harmony.

\subsection{Scalability}

Harmony is highly scalable. All the computations for a worker are carried out in the watch of the worker. So, the performance of the system is not affected as the number of workers increases, and the system can be used for almost any number of workers in an organization. Harmony also works for organizations with different numbers of dispensers, and alert zones. The system can be deployed at different sized premises, from very small to very big.

\section{DISCUSSION AND FUTURE WORK}

Standard hand hygiene guidelines [3] strongly recommend to remove watches before surgical hand hygiene preparation. So, Harmony should not be used where wearing watches are prohibited or strongly discouraged. Since a watch itself can be a germ factory due to long term use of it, watches used by the workers need to be sanitized regularly. Most of the smart watches available today are water proof, but may not be suitable for cleaning agents. It requires to be tested whether cleaning agents can be used to sanitize the smart watches. If not, the watches can be covered with a transparent and cleaning agent proof cover so that they can be sanitized.

Though we have developed a prototype of the system, it hasn't been yet deployed in real settings like hospitals or restaurants. Also, we didn't do a usability study. We plan to deploy the system at a hospital as a pilot project in the near future, and study the system performance, user acceptability, and other real world issues. In Harmony, Bluetooth enabled dispensers are used that transmits Bluetooth advertisements when soap/sanitizer is dispensed from it. Such dispensers are not commercially available now. In our prototype, we have used a smart phone app to simulate the dispense event. Before deploying the system to real settings, such dispensers need to be developed.

The devices used in most of the existing hand wash monitoring and reminder systems are dedicated for the systems only, and can not be used for other purposes. In contrast, the smart watches can be used for different purposes along with Harmony. For example, procedural reminders or organizational notifications can be provided to the workers using the smart watch. Like smart phones, many useful applications can be installed in the smart watches. We believe smart watches would serve multiple purposes in addition to hand wash monitoring and reminders.

Though Harmony is intended to be used in healthcare settings and food businesses, it can be used in settings where hand hygiene monitoring and reminder system is required. For example, social science researchers carry out research on hand hygiene practice among different groups of people such as college students $[6,23]$. The system may also be used to monitor hand wash practices, and to provide reminders to children and elderly people who often forget to wash hands.

\section{CONCLUSION}

In this paper, we present the design and implementation of Harmony, a smart watch based hand wash monitoring and reminder system. The system is designed to run on resource limited smart watches, and experimental results show that 
smart watches can be used to detect hand wash gestures, and different poses of a hand wash episode with good accuracy. The system provides reminders to workers when required, and automatically stores hand wash compliance data to a server. It is highly scalable and robust, and overcomes most of the limitations of the existing systems.

\section{Acknowledgement}

This work was supported, in part, by NSF grants CNS1239483 and CNS-1319302 and by DGIST via funding from the Ministry of Education, Science and Technology of the Republic of Korea.

\section{REFERENCES}

[1] CDC HAI prevalence survey. http://www.cdc.gov/hai/surveillance/. Last Accessed: 2014-10-07.

[2] Sony smart watch. https://play.google.com/store/devices/details? id=sony_smartwatch_3_black\&hl=en. Last Accessed: 2014-11-20.

[3] World Health Organization (WHO) guidelineson hand hygiene in health care. http://whqlibdoc.who.int/ hq/2009/who_ier_psp_2009.07_eng.pdf. Last Accessed: 2014-10-07.

[4] World Health Organization (WHO)fact sheet on healthcare associated infections. http://www. who.int/ gpsc/country_work/gpsc_ccisc_fact_sheet_en.pdf. Last Accessed: 2014-10-07.

[5] B. Allegranzi and D. Pittet. Role of hand hygiene in healthcare-associated infection prevention. Journal of Hospital Infection, 73(4):305-315, 2009.

[6] Joy L Anderson, Cynthia A Warren, Elena Perez, Reggie I Louis, Stephanie Phillips, Jean Wheeler, Melissa Cole, and Ranjita Misra. Gender and ethnic differences in hand hygiene practices among college student. American journal of infection control, 36(5):361-368, 2008.

[7] C.R. Davis. Infection-free surgery: how to improve hand-hygiene compliance and eradicate methicillin-resistant staphylococcus aureus from surgical wards. Annals of the Royal College of Surgeons of England, 92(4):316, 2010.

[8] M.B. Edmond, A. Goodell, W. Zuelzer, K. Sanogo, K. Elam, and G. Bearman. Successful use of alcohol sensor technology to monitor and report hand hygiene compliance. Journal of Hospital Infection, 76(4):364-365, 2010.

[9] C.S. Hlady, M.A. Severson, A.M. Segre, and P.M. Polgreen. A mobile handheld computing application for recording hand hygiene observations. Infection control and hospital epidemiology: the official journal of the Society of Hospital Epidemiologists of America, 31(9):975, 2010.

[10] G. Kinsella, A.N. Thomas, and R.J. Taylor. Electronic surveillance of wall-mounted soap and alcohol gel dispensers in an intensive care unit. Journal of Hospital Infection, 66(1):34-39, 2007.

[11] D.F. Llorca, I. Parra, M.A. Sotelo, and G. Lacey. A vision-based system for automatic hand washing quality assessment. Machine Vision and Applications, 22(2):219-234, 2011.
[12] Paul Martin, Bo-Jhang Ho, Nicholas Grupen, Samuel Muñoz, and Mani Srivastava. An ibeacon primer for indoor localization: demo abstract. In Proceedings of the 1st ACM Conference on Embedded Systems for Energy-Efficient Buildings, pages 190-191. ACM, 2014.

[13] S. Mohamed Rabeek, A. Norman, M. Je, M.K. Raja, R.F. Peh, and M.K. Dempsey. A reliable handwash detector for automated hand hygiene documentation and reminder system in hospitals. In The 15th International Conference on Biomedical Engineering, volume 43 of IFMBE Proceedings, pages 892-895. Springer International Publishing, 2014.

[14] S. Naikoba and A. Hayward. The effectiveness of interventions aimed at increasing handwashing in healthcare workers-a systematic review. Journal of Hospital Infection, 47(3):173-180, 2001.

[15] S. Nirjon, R. Dickerson, P. Asare, Q. Li, D. Hong, J. Stankovic, P. Hu, G. Shen, and X. Jiang. Auditeur: A mobile-cloud service platform for acoustic event detection on smartphones. In Proceeding of the 11th annual international conference on Mobile systems, applications, and services, pages 403-416. ACM, 2013.

[16] T.N. Palmore and D.K. Henderson. Big brother is washing... video surveillance for hand hygiene adherence, through the lenses of efficacy and privacy. Clinical infectious diseases, page cir781, 2011.

[17] D. Pittet, B. Allegranzi, H. Sax, S. Dharan, C. L. Pessoa-Silva, L. Donaldson, and J. Boyce. Evidence-based model for hand transmission during patient care and the role of improved practices. The Lancet Infectious Diseases, 6(10):641-652, 2006.

[18] D. Pittet, A. Simon, S. Hugonnet, C.L. Pessoa-Silva, V. Sauvan, and T.V. Perneger. Hand hygiene among physicians: performance, beliefs, and perceptions. Annals of internal medicine, 141(1):1-8, 2004.

[19] P.M. Polgreen, C.S. Hlady, M.A. Severson, A.M. Segre, and T. Herman. Method for automated monitoring of hand hygiene adherence without radio-frequency identification. Infection control and hospital epidemiology: the official journal of the Society of Hospital Epidemiologists of America, 31(12):1294, 2010.

[20] M. Ross and J. Guzewich. Evaluation of risks related to microbiological contamination of ready-to-eat food by food preparation workers and the effectiveness of interventions to minimize those risks. FDA White Paper, FDA, CFSAN, 1999.

[21] R.D. Scott. The direct medical costs of healthcare-associated infections in us hospitals and the benefits of prevention. 2009 .

[22] B. Simmons, J. Bryant, K. Neiman, L. Spencer, and K. Arheart. The role of handwashing in prevention of endemic intensive care unit infections. Infection Control and Hospital Epidemiology, pages 589-594, 1990.

[23] Cindy White, Robin Kolble, Rebecca Carlson, Natasha Lipson, Mike Dolan, Yusuf Ali, and Mojee Cline. The effect of hand hygiene on illness rate among students in university residence halls. American journal of infection control, 31(6):364-370, 2003. 